\title{
Números negativos en los siglos XVIII y XIX: fenomenología y representaciones
}

\author{
Alexander Maz Machado ${ }^{1}$ \\ Luis Rico Romero
}

\author{
${ }^{1}$ Departamento de Matemáticas, Universidad de Córdoba \\ ${ }^{2}$ Departamento de Didáctica de la Matemática, Universidad de Granada
}

\section{España}

Alexander Maz Machado. Facultad de Ciencias de la Educación, San Alberto Magno s/n. 14004-Córdoba. Spain. E-mail: malmamaa@uco.es

(C) Education \& Psychology $\mathrm{I}+\mathrm{D}+\mathrm{i}$ and Editorial EOS (España) 


\title{
Resumen
}

En este artículo se presenta una categorización sobre los fenómenos y las representaciones utilizados para presentar los números negativos en libros de texto de matemáticas publicados en España durante los siglos XVIII y XIX. Mediante un análisis de contenido en catorce textos seleccionados para el estudio, se distinguen cuatro tipologías de fenómenos: físicos, contables, temporales y matemáticos. También se identifican cuatro tipos de representaciones: verbales, numéricas, gráficas y algebraicas. Estos resultados reflejan en España un conocimiento y tratamiento de los números negativos al mismo nivel que el de otros países europeos de la época.

Palabras clave. Números negativos, fenomenología, representaciones, libros de texto, historia de las matemáticas.

Recibido: 14/11/08 Aceptación inicial: 17/11/08 Aceptación final: 23/01/09

\begin{abstract}
In this article is displayed a categorisation on the phenomena and the representations used to introduce the negative numbers in mathematics books published in Spain during the XVIIIth and XIXth centuries. Through a content analysis of the fourteen texts selected to the study we distinguished four typologies of phenomena: physical, countable, temporal and mathematical. Four types of representations are also identified: verbal, numeric, graphic and algebraical. These results reflects that the knowledge and treatment of negative numbers in Spain were at the same level of other European countries in the studied period.
\end{abstract}

Keywords. Negative numbers, phenomenology, representations, textbooks, mathematics history.

Received: 11/14/08 Initial Acceptance: 11/17/08 Final Acceptance: 01/23/09 


\section{Historia de las Matemáticas y Educación Matemática}

La Educación Matemática se dedica a estudiar cómo las personas aprenden y hacen matemáticas en todos los niveles y también cómo ese aprendizaje es influenciado por las estrategias de enseñanza (Dörfler, 2000; Schoenfeld, 2000) y, puesto que la Matemática tiene una larga historia, también es importante investigar de qué forma los conocimientos matemáticos fueron evolucionando a lo largo del tiempo hasta adquirir los significados científicos y desarrollos formales aceptados en el actual momento cultural. Al respecto, Wussing (1998) sostiene que sin la historia de los conceptos, de los problemas y de disciplinas matemáticas especiales, el cuadro de desarrollo de la Matemática quedaría incompleto, puesto que todo conocimiento o idea matemática se ha gestado en una situación histórico-social concreta.

Algunos conceptos matemáticos presenta una complejidad y unas determinadas características de abstracción que han hecho que su aceptación, desarrollo y formalización fuese no solamente lento si no que también generaron vigorosas y tajantes diferencias epistemológicas entre algunos prestigiosos matemáticos en la época de su emergencia y consolidación. Una rápida inspección revela al número negativo como un claro ejemplo de uno de los conceptos históricamente polémicos.

Las dificultades epistemológicas generadas por el número negativo aún tienen vigencia cuando en la práctica cotidiana de la enseñanza de las Matemáticas, los docentes encuentran cierta resistencia o dificultad en los alumnos para llegar a su comprensión, interpretación y utilización correctas. Los números negativos han llamado la atención de los investigadores en Educación Matemática dado que las dificultades manifiestas en los alumnos para su entendimiento repercuten tanto en la comprensión de otros conceptos como en la correcta interpretación de determinadas situaciones y la solución de problemas asociados a ellas (Maz y Rico, 2007).

Algunos autores afirman que las dificultades de los alumnos para comprender y operar con los números negativos reflejan las etapas del desarrollo histórico de estos números (Glaeser, 1981; González Marí, 1995), de tal manera que se hace necesario conocer un poco sobre su historia para llevar a cabo su enseñanza. Como afirma Gómez (1993; p. 13): “Conocer la evolución histórica de los sistemas de numeración y saber 
las razones que provocaron los cambios y el abandono de unos sistemas por otros, contribuye a dar sentido a los conocimientos previos o ya adquiridos". Al respecto, es claro que el propósito fundamental de la historia es el "estudio general del cambio a través del tiempo, esto es altamente relevante no únicamente para las matemáticas que estudiamos a diario, sino para la comunicación de las matemáticas a todos los niveles" (Rogers, 1993; p. 107).

\section{Libros antiguos de Matemáticas: fuentes primarias para la indagación didáctica}

En la construcción de los conceptos en un marco científico, particularmente en el conocimiento matemático, el lenguaje asume un importante papel mediador, donde su referente es el lenguaje textual. Como afirma Lizcano (1993, p. 30), es en el texto donde "efectivamente se producen las matemáticas", con lo cual cobra importancia epistemológica el análisis de los libros de texto de Matemáticas.

Cuando se trabaja en Educación Matemática y se estudian libros de texto, hay que considerar que los textos que se estudian y analizan son fuentes primarias para conocer el estado del conocimiento científico de una época, así como para estudiar el modo en que estos conocimientos se incardinaban en los planes de formación vigentes en el momento de su publicación. Los textos son documentos didácticos ubicados en determinado marco curricular y, por tanto, el análisis de su contenido ha de contemplar la naturaleza didáctica de los documentos. Desde esta perspectiva, subrayamos que los textos de matemáticas no son documentos exclusivamente formales, sino que son materiales docentes, con propósitos educativos, que se proponen transmitir unos determinados significados para la correcta comprensión de los conceptos formales que presentan (Segovia y Rico, 2001).

Estas ideas señalan que el estudio de los libros de texto, actuales o del pasado, aporta nuevas ideas para la comprensión del desarrollo, tanto de los conocimientos específicos de una ciencia, como de los planes curriculares y de los sistemas educativos de un país.

Consideramos que esta necesidad e interés por la historia y el desarrollo de los conceptos avala en cierta medida la pertinencia de la investigación que realizaremos 
sobre una parte relevante del análisis de contenido, la relativa al estudio de la fenomenología y representaciones de los números negativos. Así contribuimos a sustentar que "el conocimiento de la propia historia es uno de los estímulos más fuertes para consolidar la propia identidad” (Rico y Sierra, 1994; p. 100).

\section{Descripción del estudio}

En este documento centramos la atención en los libros de texto para las Matemáticas en España en los siglos XVIII y XIX, especialmente en aquellos aspectos de sus contenidos antes señalados. Si bien en este período los matemáticos españoles no destacan por sus contribuciones al desarrollo de las Matemáticas, sí se produce una importante actividad matemática, que estuvo centrada tanto en la enseñanza como en la aplicación y en la difusión de los conocimientos por medio de la publicación de libros (Rico y Maz, 2005).

Las dos preguntas que orientan este estudio son:

¿Qué fenómenos se utilizan para ejemplificar y justificar la introducción de los números negativos en los libros de matemáticas durante los siglos XVII y XIX en España?

¿Cómo se representan los números negativos en los libros seleccionados?

Durante los siglos XVIII y XIX en España se presentan grandes contrastes tanto en el tipo de libros de texto de Matemáticas que se escriben, en el prototipo de los autores, así como en la clase social de sus destinatarios (Maz, 2005). Por ello hemos seleccionado algunos textos significativos de Aritmética y Álgebra, representativos de este período histórico.

Los criterios seguidos para seleccionar los libros fueron:

a) que se hubiesen publicado en España entre 1700 y 1900;

b) que su autor fuera español;

c) que estuvieran escritos en español.

Se revisaron 64 textos y finalmente se eligieron 15 para el estudio (ver el listado anexo). 
La representatividad de los autores de libros de matemáticas selecccionados para el estudio es, principalmente, cultural, y permite mostrar la presencia de ideas en la producción matemática del periodo analizado.

\section{Fenomenología y Representaciones}

Si consideramos los organizadores curriculares que propone Rico (1997), vemos que la mayoría de ellos pueden ser localizados en los libros de texto de matemáticas y en particular en los antiguos, al tratarse de documentos curriculares que tienen una estructura y una organización determinadas por su carácter educativo. Centraremos la atención en dos de estos organizadores:

- los fenómenos que están en el origen de los conceptos, y

- los modos en que tales conceptos se hacen presentes, es decir, sus representaciones.

La fenomenología de una estructura matemática estudia los fenómenos que están en la base de cada uno de los conceptos que la caracterizan. La fenomenología, o análisis fenomenológico, considera los problemas o cuestiones básicas a las que da respuesta un determinado concepto matemático, así como las situaciones usuales en las que estas cuestiones se plantean (Rico, Lupiáñez, Marín y Gómez, 2008). Estos fenómenos, con mayor o menor amplitud, están en la base de los ejemplos y actividades que se presentan en un libro de texto. Por tal razón, si se quiere presentar un tópico matemático en toda su riqueza conceptual y procedimental, mostrando la pluralidad de sus diversos usos y significados, debe considerarse mediante una diversidad de contextos y situaciones, en conexión con diferentes problemas, y debe conectarse con otros campos diferentes del conocimiento (Rico y Lupiáñez, 2008).

En los procesos de aprendizaje de las Matemáticas la fenomenología ocupa un lugar destacado porque el pensamiento matemático surge de los fenómenos que las estructuras matemáticas abstraen y organizan, fenómenos procedentes de grandes familias de los mundos natural, social y mental (Rico et al., 2007).

Puig (2001) señala que la fenomenología es un medio para organizar las ideas matemáticas y, cuando estas ideas están relacionadas con los sistemas escolares en los 
que se pretenden enseñar, estamos ante la fenomenología didáctica.

Los autores estudiados justifican la introducción de los números negativos de diversas formas, desde la interpretación de situaciones concretas tales como desplazamientos, hasta la ampliación formal de la sustracción, pasando por interpretaciones operativas, y explicaciones retóricas propias de la Aritmética.

Las situaciones que se utilizan para ejemplificar y caracterizar las cantidades negativas se organizan de manera global en cuatro grupos:

- fenómenos físicos,

- situaciones contables,

- situaciones temporales o cronológicas, y

- contextos matemáticos.

A continuación se identifican estas situaciones y se presenta un ejemplo de cada una de ellas:

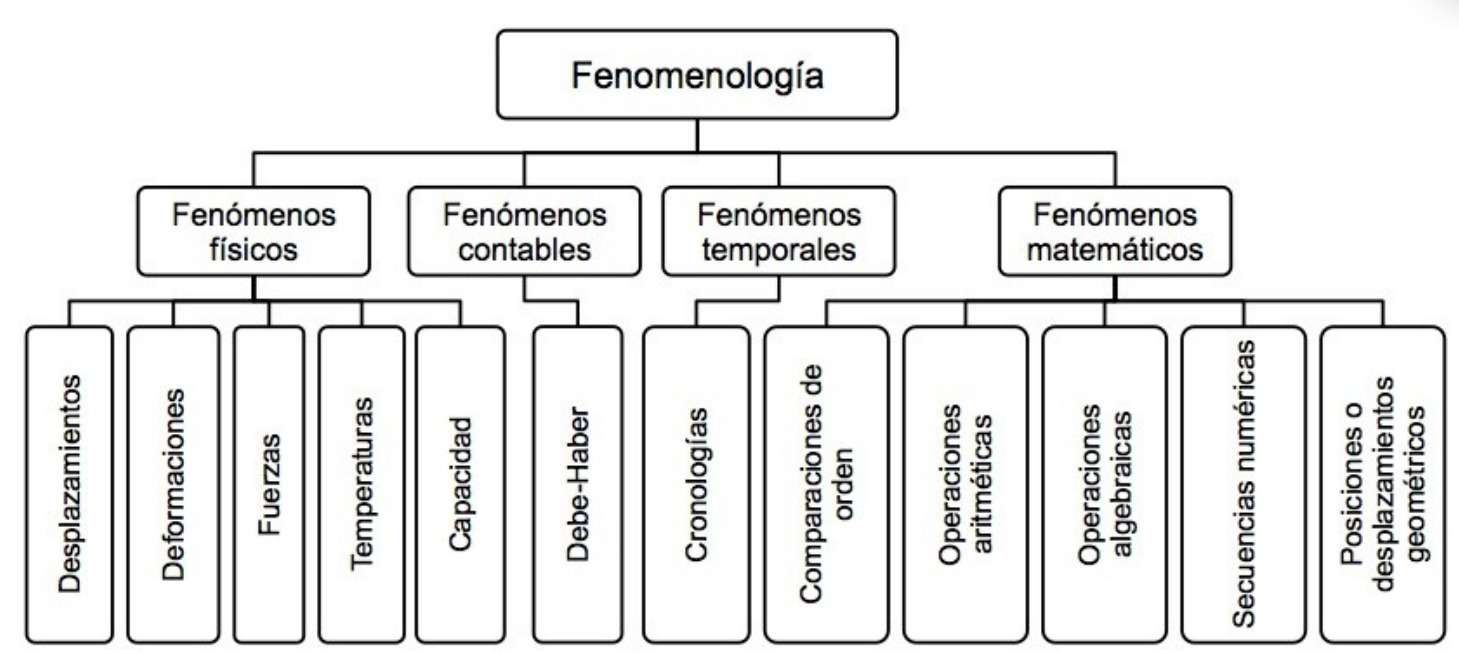

Figura 1. Tipologías fenomenológicas

\section{Fenómenos físicos}

Los distintos autores recurren con frecuencia a fenómenos que se dan en la naturaleza y son explicados mediante leyes físicas; parece que éstos facilitan la introducción de los números negativos en situaciones relativas familiares para los 
alumnos. Se encuentran ejemplos de cinco clases de ellos.

a) Desplazamientos: Se apoyan en situaciones de avances o retrocesos de objetos. Éstos son comparaciones de medidas dirigidas con sentidos opuestos. Se utilizan las cantidades negativas para modelizar un fenómeno de movimiento en retroceso:

"Es facil formar ideas de eftas Cantidades menores que nada. Supóngafe, que defde C. hasta B. ay tres leguas; y defde C. hasta A. ay dos. Si vn Caminante efta en C. con defignio de llegar a B. y effectivamente faliendo de C. llega a B. es verdad dezir, que abançó ; y que fu abance es mayor que nada; y que la medida de efte abance es tres leguas. Si no obftante su defignio fe halla precisado a detenerse en C. su abance es ninguno, o es igual a nada. Si en vez de caminar azia B. saliendo de C. llega a A. en la phrase vulgar fe dira, que cejò ; y para expressar, que hizo lo contrario, de lo que avia de hacer, puede dezirse, que ha abançado menos que nada, y que fu avance es, -2. leguas: conque aquí, dos leguas fon menos que nada." (Ulloa, 1705, p. 20)

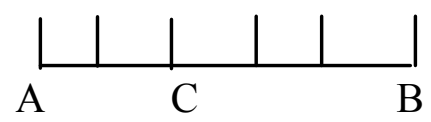

Este ejemplo presenta una comparación de situaciones que llevan a comparaciones mediante sentidos opuestos. Se fundamenta en la medida de distancias con direcciones diferentes y contrarias. El origen o punto de partida $\mathrm{C}$ lo utiliza como punto de referencia para los dos avances y para el intento de avance. Como la escogencia de sentidos es arbitraria, la medida obtenida como negativa podría ser -2 o -3 según la conveniencia del resolutor. Corresponde a un fenómeno cotidiano basado en los desplazamientos en el que se asigna a la cantidad negativa una naturaleza de acción; considera una cantidad negativa como resultado de una transformación o cambio.

b) Deformaciones: Presentan contextos en los que algunos objetos son sometidos a una determinada acción en una u otra dirección.

“[...] por último si a expresa lo que se ha alargado una linea, -a representará lo que se ha acortado" (Verdejo, 1794; p. 37). 
Se recurre a una situación donde el objeto cambia su estado inicial y sufre algún tipo de variación en cuanto a su longitud.

c) Fuerzas: Se presentan analogías entre la acción y reacción de la tercera ley de Newton y las cantidades positivas y negativas.

“[...] si a es el valor de una fuerza que obra de derecha á izquierda, -a será la misma fuerza obrando de la izquierda ácia la derecha" (Juan Justo García, 1782; p. 54).

Es interesante ver cómo el autor utiliza la tercera ley de Newton (acción y reacción en la Física Dinámica) para ilustrar la naturaleza de las cantidades negativas, aunque en rigor se está refiriendo a magnitudes dirigidas más que a números enteros.

d) Temperaturas: Las variaciones térmicas medidas en el termómetro respecto al valor del cero se utilizan para compararlas con el paso de valores positivos a los negativos a lo largo de la recta numérica.

"[...] para apreciar los grados de temperatura, no sólo conviene saber cuántos son; sino tambien si están por encima ó por bajo del cero del termómetro" (Fernández y Cardín, 1858; p. 10).

La situación del termómetro y las temperaturas, es una situación que perfectamente se representa en una modelización a través de los números enteros por cuanto se identifica que hay un cero único y es coherente pasar de un lado del cero al otro por que se sigue indicando el valor de un mismo tipo de cantidades, esto es temperaturas.

e) Capacidad: La entrada y salida de productos o sustancias en lugares o recipientes sirve para suscitar símiles con los números negativos y positivos al efectuarse una operación matemática sobre ellos.

“[...] si nos proponemos averiguar en quanto tiempo se llenará un estanque de agua, en que por un lado entra agua y por otro sale, tendremos que atender no solo al agua 
que entra, sinó tambien al agua que sale; y como el agua que entra conspira al fin que nos proponemos, esta será la positiva ; y la que sale, conspira á vaciar el estanque, que es lo contrario de llenarle, será la negativa" (Vallejo, 1813; p. 163, segunda edición).

\section{Fenómenos contables.}

Estos fenómenos están relacionados con el manejo de capitales mediante la relación debe-haber o deudas y ganancias; sirven tanto para dar significado a las situaciones negativas como para ilustrar lo que entienden los autores por una cantidad menor que nada.

\footnotetext{
"Figurémonos ahora que ajustamos las cuentas á otro sugeto, y encontramos que se empeña en 300 ducados cada año; si queremos comparar la situación de estos dos sugetos, con el fin de averiguar el que mas se empeña, diremos que este último: porque es mucho mayor 300 ducados que 150 ducados que sacábamos antes. Pero si suponemos la qüestión resuelta por Álgebra, con el fin de buscar el ahorro de este sugeto, encontraríamos que su ahorro anual sería -300 ducados; y si quisiéramos comparar ese ahorro con el anterior, que era -150 , no diríamos que -300 sea mayor ahorro que -150, sino al contrario; porque en un sentido absoluto, si buscamos cual de los dos ahorra mas, y encontramos que ninguno ahorra, el que tiene el estado mas ventajoso es aquel que menos se empeña" (Vallejo, 1813; pp. 167-168).
}

Hay una situación cotidiana con cantidades relativas (tener/deber), para dotar de significado numérico a las cantidades negativas. En este caso la comparación de capitales es el fenómeno que se modeliza por medio de las cantidades negativas

\section{Fenómenos temporales.}

Se refieren a la comparación de un período o época respecto a una fecha determinada por un hecho singular, como por ejemplo lo pueden ser el nacimiento de Cristo o la Revolución Francesa. 
“[...] el tiempo anterior y posterior á una época determinada [...], son cantidades directamente contrarias, cuya diferente acepción, si bien dependiente en gran parte de la Voluntad del calculador, necesita indicarse con signos representativos del carácter positivo y negativo, en que consiste su contrariedad" (Fernández Vallín y Bustillo, 1857; p. 233).

\section{Fenómenos matemáticos.}

Se recurre a los objetos del mundo matemático para ilustrar los negativos. Se manifiesten cinco clases de ellos:

a) Comparaciones de orden: A través de comparaciones entre valores numéricos.

“[...] Tambien dada $-5<-3$, sucede otro tanto en $-5+2<-3+2$ y $-5-2<-3-2$. Lo mismo en $-3<2$ y $-3+4<2+4$ y $-3-4<2-4$." (Odriozola, 1827p. 444).

b) Operaciones aritméticas: Mediante adiciones o sustracciones.

"Si un politivo +3, fe fumma con vn privativo, ò igual à él, -3. ò mayor que él -4. ò menor que èl, -2 la fumma ferà $+3-3$. ò , +3-4. ò, +3-2:porque la fumma fera +3+-3. ò, +3+-4. ò, +3+-2 [...]” (Ulloa, 1705; p. 21).

c) Operaciones algebraicas: Se recurre a la extracción de raíces de expresiones algebraicas y a la resolución de problemas.

"Sea yy $+5 y+6 \Omega 0$. Pidese su solucion. Los divisores del vltimo termino son los mismos que en la pasada; porque todas las raíces han de ser negativas, por no aver alternación de signos, intentarè la partición por $\mathrm{y}+2 \Omega 0$. por y $+3 \Omega 0$. \&c. Y porque hallo venir justa por y $+2 \Omega 0$. digo, que vna raiz es -2 . y la otra -3 " (Tosca, 1707; p. 189).

d) Secuencias numéricas: Las sucesiones y series permiten observar el posicionamiento de los valores numéricos según su signo y valor.

"Se llama progresión creciente aquella en que la razon es positiva y los términos van por tanto aumentando; y decreciente en el caso contrario. [...] 
$\div 16,12,8,4,0,-4,-8, \ldots$ (Fernández y Cardín, 1858; p. 113).

e) Posiciones o desplazamientos geométricos: La recta numérica y los desplazamientos sobre ella, así como las traslaciones y la rotación de los segmentos alrededor de un eje puntual o sobre la circunferencia, permiten "explicar" a algunos autores el cambio de signo en los números. De esta manera el segmento cambia su posición sin variar su magnitud.

"Ni tampoco es razon bastante la que se deduce de la série natural aritmética

$$
\div \ldots .-6,-5,-4,-3,-2,-1,0,1,2,3,4,5,6, \ldots .
$$

á la cual erróneamente atribuye Mr. Coyteux el carácter de creciente, cuando no es sino ascendente, explicándose el ascenso uniforme de ella por el decremento cuantitativo de los términos negativos hasta cero, y el incremento de los positivos. Este ascenso progresivo podria representarse de una manera geométrica, haciendo perpendiculares por bajo y por encima de un eje horizontal los valores absolutos ó numéricos de todos los términos: una oblícua que pasase por el punto de orígen, sería el lugar geométrico de todas estas longitudes"

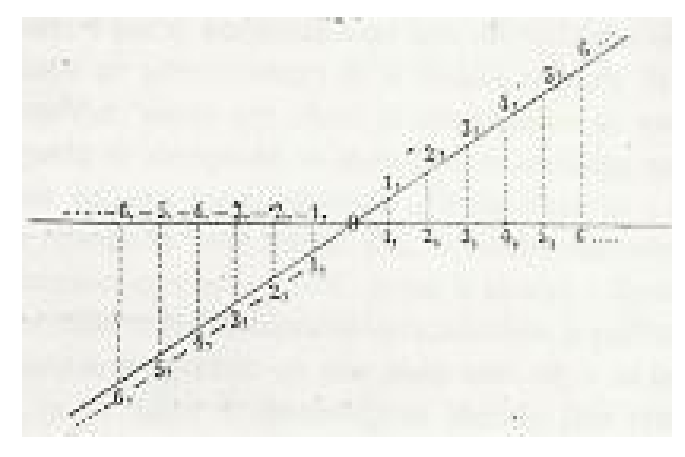

Figura 2.

(Rey y Heredia, 1865; p. 33)

\section{Sistemas de representación.}

Los conceptos se muestran mediante distintos tipos de símbolos, gráficos o signos, y cada uno de ellos constituye una representación del concepto en cuestión (Castro y Castro, 1997). Los sistemas de representación permiten al autor hacer presentes y comunicar a los lectores o alumnos las ideas matemáticas que quiere transmitirles, en nuestro caso tenemos acceso a las representaciones escritas. 
El análisis de los textos de Matemáticas seleccionados ha permitido identificar cuatro tipos de representaciones, utilizadas por sus autores:

a) Verbales: el autor recurre a dar explicaciones sobre los números negativos mediante descripciones verbales con una alta carga retórica.

"Supongase, que un hombre no tiene bienes algunos, y que debe 1000. escudos; y otro hombre no tiene tampoco bienes algunos, pero no debe nada; es cierto tiene el primero peor fortuna que el segundo; pero este tiene nada: luego el primero tiene menos que nada. Tambien, si al que no tiene bien alguno, y debe 1000. escudos, le dàn 1000. escudos, con que paga la deuda, aumenta a sus bienes; pero sus bienes aun después de ese aumento son nada; luego antes del aumento, sus bienes eran menos que nada" (Tosca, 1709; p. 92-93).

b) Numéricas: sólo se utilizan combinaciones de números y signos para dar idea y explicar las cantidades negativas. Todos los autores utilizan notaciones numéricas para presentarlas.

"Serie entera. Si se colocan en fila todos los números positivos y negativos, á contar desde el cero, la serie

$$
\ldots .-5,-4,-3,-2,-1,0,1,2,3,4,5, \ldots \ldots
$$

será el lugar ó dominio de todas las sumas enteras, de manera que los sumando y las sumas se hallarán á la vez en la misma serie" (García de Galdeano, 1883; p. 48).

c) Gráficas: se recurre a la gráfica por medio de una recta, la cual contiene generalmente letras que indican lugares o posiciones. Aunque el esquema gráfico es el mismo en varios autores, si comparamos la representación gráfica de Ulloa en 1706 con la de Cortázar en 1892, es prácticamente la misma, sin embargo varían los argumentos utilizados para justificar la cantidad negativa que surge de allí. 


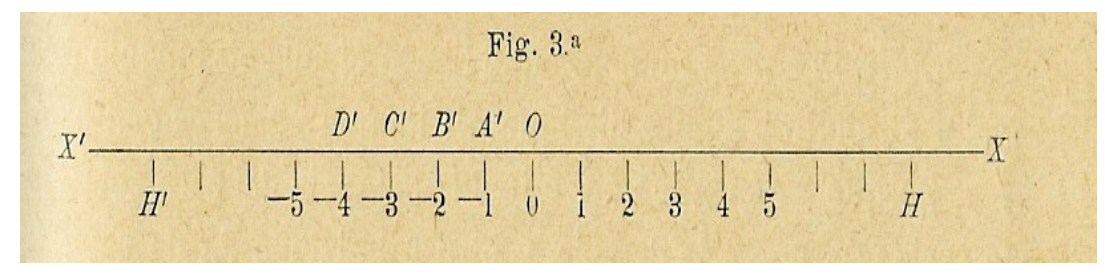

Figura 3.

(Octavio de Toledo, 1900; p. 81).

d) Algebraicas: estas representaciones combinan los números con los signos y las letras; se utilizan las ecuaciones como recurso para mostrar cómo surgen y se operan las cantidades negativas.

“[...] hemos supuesto implícitamente que la operación directa $\cap$ verificada sobre una serie de objetos

$$
\mathrm{a}, \mathrm{b}, \mathrm{c}, \mathrm{d} \ldots,
$$

era siempre posible, y daba por resultado un objeto perteneciente á esa misma serie, que llamaremos serie directa; suponíamos además que constituida una nueva serie con los objetos recíprocos de los de la serie (A), la serie

$$
\bar{a}, \bar{b}, \bar{c}, \bar{d}, \ldots . ., \quad(\bar{A})
$$

que llamaremos serie recíproca;" (Octavio de Toledo, 1900; pp. 52-53)

\section{Conclusiones}

Los fenómenos que están en la base del sistema de los números negativos son aquellos que se basan bien en la comparación de cantidades, utilizando las relaciones "más que" o "menos que"”, o bien en transformaciones entre cantidades, que expresan incremento o disminución de una cantidad de partida, lo que permite comparar las situaciones inicial y final con los comparativos "mayor que" o " menor que".

En los libros analizados se observa que los autores utilizan fenómenos a partir de contextos y situaciones que proceden tanto de su entorno cotidiano como de la Matemática.

En nuestro estudio hemos localizado cuatro tipos de situaciones utilizadas por 
los autores españoles de libros de texto de matemáticas de los siglos XVIII y XIX. Estas situaciones, como hemos ejemplificado corresponden a: fenómenos físicos, situaciones contables, situaciones temporales o cronológicas, y contextos matemáticos.

Los fenómenos físicos localizados corresponden a desplazamientos, deformaciones, fuerzas resultantes, temperaturas y capacidades. En todos estos casos hay una trasformación de cantidades que suponen un aumento o una disminución de una cantidad inicial, lo cual permite expresar el cambio mediante un valor positivo o negativo. Hay por tanto dos tipos de cantidades: las que producen aumento (positivas) y las que producen disminución (negativas). Se trata en este caso de lo que Kant denomina "cantidades adjetivadas". Los números negativos expresan en este caso cantidades relativas, con una estructura de doble número natural, es decir números naturales con direcciónes contrarias, siendo estas distintas de los números enteros.

En el caso particular de la temperatura se considera un punto inicial de equilibrio (punto cero o punto de congelación) que se toma como valor cero, respecto al cual las cantidades que incrementan ese valor son positivas y las que lo disminuyen son negativas, estableciéndose una continuidad entre valores negativos y positivos. Se trata de números relativos, con una relación de orden que admite comparaciones entre la parte negativa y la positiva.

Los fenómenos contables muestran un uso de los negativos en una doble contabilidad de haber/deuda, que trabaja con cantidades adjetivadas, al igual que en los fenómenos físicos, ejemplificando de esta foma números relativos.

Los fenómenos temporales, al igual que en el caso de la temperatura, permiten fijar un punto de referencia, a partir del cual los avances son positivos y los retrocesos son negativos, con continuidad entre cantidades positivas y negativas.

En los fenómenos matemáticos se pone en juego la estructura algebraica que procede de la ecuación $a+x=b$, y se obtienen las implicaciones de dar valor numérico a su solución en el caso en que $b<a$. Los números negativos con los que se trabaja en estas situaciones son números enteros.

Todas estas situaciones muestran contextos de aplicación de los números 
negativos y ponen de manifiesto la estructura matemática que subyace en la interpretación de este concepto en cada caso. La variedad de fenómenos hallados revela el interés y la preocupación de los autores de texto de los siglos XVIII y XIX por presentar, explicar y aplicar el concepto de número negativo; también muestran las distintas concepciones que subyacen en el concepto de número negativo.

La evolución de los sistemas de representación refleja la evolución en la comprensión de los números negativos y como su formalización va tomando su lugar en los libros de Matemáticas como lo revelan las representaciones halladas en el estudio: verbales, numéricas, gráficas y algebraicas.

El análisis de los antiguos manuales de Matemáticas arroja valiosa información sobre el largo camino que siguieron los números negativos hasta que se formalizan y aceptan en el mundo matemático; estos resultados pueden llegar a ser contrastados con los fenómenos y representaciones actualmente utilizados en los manuales escolares de Matemáticas, por lo que cobra interés su estudio.

\section{Referencias}

Castro, E. y Castro E. (1997). Representaciones y modelización. En L. Rico (Coord.): La Educación Matemática en la Enseñanza Secundaria. (pp. 95-124). Barcelona: Horsori.

Dörfler, W. (2000). Mathematics, mathematics education and mathematicians: An unbalanced triangle. International Commission on Mathematical Instruction. http://www.emis.de/mirror/IMU/ICMI/bulletin/49/Mathematics.html

Glaeser, G. (1981). Epistémologie des nombres relatifs. Recherches en Didactique des Mathématiques, 2(39), 303-346.

Gómez, B. (1993). Numeración y cálculo. Madrid: Síntesis.

González Marí, J. L. (1995). El campo conceptual de los números naturales relativos. Tesis Doctoral. Universidad de Granada.

Lizcano, E. (1993). Imaginario colectivo. La construcción social del número y el infinito. Madrid: Paidós.

Maz, A. (2005). Números negativos en España en los siglos XVIII y XIX. Tesis doctoral, Universidad de Granada, Granada. 
Maz, A. y Rico, L. (2007). Situaciones asociadas a los números negativos en textos de matemáticas españoles de los siglos XVIII y XIX. PNA, 1(3), 113-123.

Puig, L. (2001). Notas para una lectura de la fenomenología didáctica de Hans Freudental. En H. Freundental: Fenomenología didáctica de las estructuras matemáticas. (textos seleccionados). Segunda edición. México: CINVESTAV.

Rico, L. (1997). Los organizadores del currículo de matemáticas. En L. Rico (Coord), La educación matemática en la enseñanza secundaria. (pp. 39-59). Barcelona: ice-Horsori.

Rico, L. y Lupiáñez, J. L. (2008). Competencias matemáticas desde una perspectiva curricular. Madrid: Alianza Editorial.

Rico, L., Lupiáñez, J. L., Marín, A. y Gómez, P. (2007). Matemáticas escolares y análisis de contenido con profesores de secundaria en formación. Trabajo presentado en VIII Seminario de Investigación Pensamiento Numérico y Algebraico (PNA) de la SEIEM. Aravaca.

Rico, L. y Maz, A. (2005). Matemáticas, libros y matemáticos: un recorrido por su historia y su relación con la enseñanza en España. En M. Torralbo (Ed.), El libro español de Matemáticas (pp. 11-35). Córdoba: Servicio de Publicaciones de la Universidad de Córdoba.

Rico, L. y Sierra, M. (1994). Educación matemática en la España del Siglo XX. En Kilpatrick, J., Rico, L. y Sierra, M. Educación Matemática e Investigación. Madrid: Síntesis.

Rogers, L. (1993). The historical construction of mathematical knowledge. En Lalande, F., Jaboeuf, F., \& Nouazé, Y. (eds.) History and epistemology in mathematics education. First european summer university proceedings. (pp. 105-112). Montpellier. IREM de Montpellier.

Schoenfeld, A. H. (2000). Purposes and methods of research in mathematics education. Notice of AMS, 47(6), 641-649.

Segovia, I. y Rico, L. (2001). Unidades didácticas. Organizadores. En E. Castro (ed): Didáctica de las matemáticas en la educación primaria. (pp. 83-104). Madrid: Síntesis.

Wussing, H. (1998). Lecciones de historia de las matemáticas. Madrid: Siglo XXI.

\section{Listado cronológico de los libros de texto de matemáticas utilizados}


Pedro de Ulloa (1706). Elementos Mathematicos. Tomo I. Madrid: Antonio González de Reyes, Impresor.

Thomas Vicente Tosca (1727). Compendio Matemático. Tomo II. Segunda edición corregida y enmendada. Madrid: Imprenta de Antonio Marín.

Thomas Cerdá (1758). Liciones de Mathematica, o Elementos Generales de Arithemtica y Algebra para el uso de la clase. Tomos I y II. Barcelona: Francisco Suriá, Impresor de la real Academia de Buenas Letras de dicha ciudad.

Benito Bails (1772). Elementos de Arismética. Tomos I y II. Primera edición. Madrid: D. Joaquín Ibarra. Impresor de la cámara de S.M.

Juan Justo García (1782). Elementos de Aritmética, Álgebra y Geometría. Quarta edición. Tomo primero. Salamanca: Imprenta de D. Vicente Blanco.

Francisco Verdejo González (1794). Compendio de Matemáticas puras y mixtas. Tomo primero. Madrid: Imprenta de la viuda de Ibarra.

José Mariano Vallejo (1813). Tratado elemental de matemáticas. Tomo I. La Aritmética y álgebra. Segunda edición. Madrid: Imprenta Garrasayaza.

José de Odriozola (1844). Curso completo de Matemáticas puras. Tomo I reformado. Aritmética y Álgebra elemental. Tercera edición. Madrid: Imprenta de los señores viuda de Jordán é hijos.

Jacinto Feliu (1847). Tratado elemental de matemáticas. Para el uso del Colegio General Militar. Tomo II. Álgebra. Madrid: Imprenta de D. José M. Gómez Colón y Compañía.

Acisclo Fernández Vallín y Bustillo (1857). Elementos de Matemáticas. Aritmética y Álgebra. Geometría, Trigonometría y Nociones de Topografía. Nueva edición estereotípica. Madrid: Imprenta de la Viuda de Hernando y Compañía.

Joaquín Maria Fernández y Cardín (1884). Elementos de matemáticas. Algebra Decimocuarta edición notablemente mejorada. Madrid: Imprenta de la viuda e hija de Fuentenebro.

José María Rey y Heredia (1865). Teoría transcendental de las cantidades imaginarias. Madrid: Imprenta Nacional.

Zoel García de Galdeano y Yaguas (1883). Tratado de Álgebra. Tratado Elemental. Parte primera. Madrid: Imprenta de Gregorio Juste.

Luis Octavio de Toledo (1900). Elementos de aritmética universal. Calculatoria. Madrid: Imprenta Fortanet. 